\title{
A rare but devastating cause of cardiac tamponade
}

\author{
R. S. Hermanides · A. H. Tavenier · A. P. Nierich
}

Published online: 9 May 2019

(c) The Author(s) 2019

A 37-year-old male patient was admitted to hospital with a 1-month history of progressive dyspnoea. He had no cardiac history. Cardiovascular examination was highly suspicious for cardiac tamponade. Transthoracic echocardiography and computed tomography (CT) of the chest showed an extra cardiac mass and $6 \mathrm{~cm}$ pericardial effusion with compression of the right atrium and ventricle (Fig. 1a). Emergency surgery was performed. There was a haemopericardium due to active bleeding of a fistula between the right coronary artery (RCA) and a large mass $(15 \times 6 \mathrm{~cm})$ that invaded into the pericardium and epicardium. The mass and a large part of the pericardium were removed and a pericardial patch was placed. As the mass protruded into the coronary artery, revascularisation of the RCA could not be performed. Because of refractory cardiogenic shock due to right ventricular failure the patient could not be weaned from extracorporeal circulation. The patient died on the intensive care unit shortly after surgery. Pathological examination revealed an angiosarcoma (Fig. 1b).

Conflict of interest R.S. Hermanides, A.H. Tavenier and A.P. Nierich declare that they have no competing interests.

Open Access This article is distributed under the terms of the Creative Commons Attribution 4.0 International License (http://creativecommons.org/licenses/by/4.0/), which permits unrestricted use, distribution, and reproduction in any medium, provided you give appropriate credit to the original author(s) and the source, provide a link to the Creative Commons license, and indicate if changes were made.

Fig. 1 a Chest CT image with a very large extracardiac mass and $6 \mathrm{~cm}$ pericardial effusion with compression of the right atrium and right ventricle. Space-occupying lesion in the right atrium, diffuse lesion in both lungs and in the spleen. Pleural effusion right. b Macroscopic image of an angiosarcoma
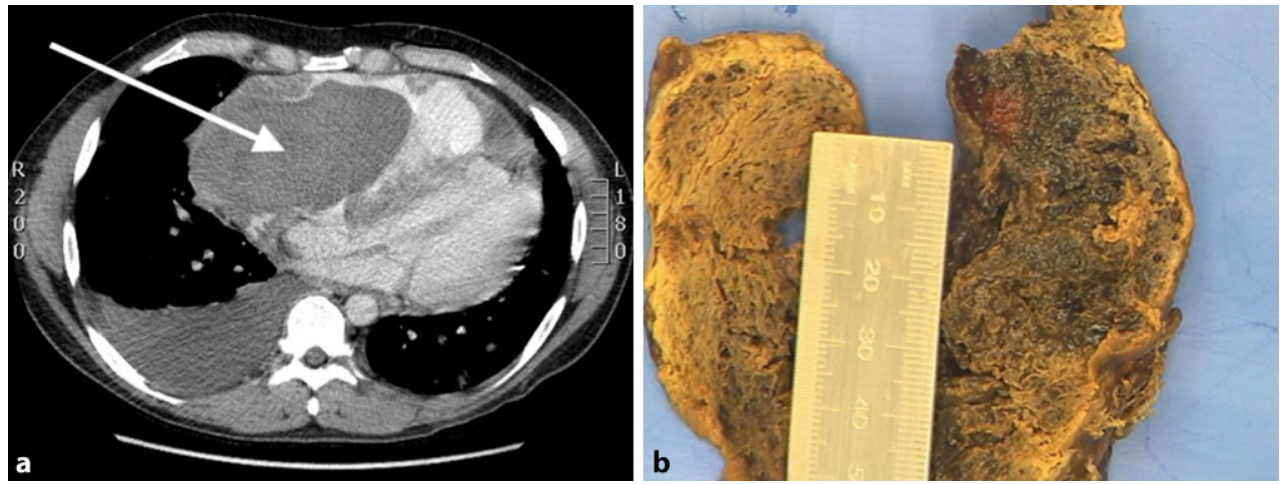

R. S. Hermanides $(\bowtie) \cdot A$. H. Tavenier

Departments of Cardiology, Isala Hospital, Zwolle, The

Netherlands

r.s.hermanides@isala.nl

\section{A. P. Nierich}

Department of Cardiothoracic Intensive Care and OR, Isala

Hospital, Zwolle, The Netherlands 